\title{
Management of HIV and AIDS at lower primary health care facility in Chalinze, eastern Tanzania
}

\author{
BONIPHACE IDINDILI ${ }^{1,2^{*}}$, BONIFACE JULLU ${ }^{3}$, FERDNAND M. MUGUSI ${ }^{4}$ and MARCEL TANNER ${ }^{1}$ \\ ${ }^{1}$ Swiss Tropical and Public Health Institute, University of Basel, Socinstrasse 57, 4002, Basel, Switzerland \\ ${ }^{2}$ Tumbi Special Hospital, P.O Box 30041, Kibaha, Tanzania \\ ${ }^{3}$ Ifakara Health Institute, P.O Box 53, Ifakara, Tanzania \\ ${ }^{4}$ Muhimbili University of Health and Allied Sciences, School of Medicine, Dar es Salaam, Tanzania
}

\begin{abstract}
Implementation of Antiretroviral Therapy (ART) services at health centres in Tanzania were delayed due to several reasons including shortage of qualified staff, inadequate infrastructure and logistics problems. However, patients from peripheral areas experienced difficulties in accessing ART services due to long distances from clinics. National AIDS Control Programme (NACP) and NonGovernmental Organizations (NGOs) embarked on ART services scale-up programme aimed at improved ART availability and accessibility. Through this programme ART services were established at health centres and selected dispensaries. However, no previous documented experiences existed at country level to guide provision of services. Therefore, this study was designed to gather experiences and share lessons learnt with other health care providers and programme implementing partners. This was a descriptive cross-sectional study involved patients enrolled to ART services between May 2007 and April 2009. Data collection involved observation of health providers' performance and retrospective ART and care patients' registers review. During the study period, 611 care and 284 ART patients were attended. Majority of patients (85.1\%; 762/895) were adults aged 25-45 years. In total 61.5\% (550/895) of the patients had CD4+T lymphocytes $\leq 350 / \mu \mathrm{l}$ the cut-off point for initiating ART. The frequency of symptoms was noted to significantly decrease with increasing CD4 counts $(P<0.001)$. Numbness, parotid enlargement and genital discharge were not related to patient level of CD4+T-lymphocytes counts. Papular pruritic eruptions $98 / 282(34.8 \%)$, tuberculosis $86 / 282(30.5 \%)$ and oesophageal candidiasis $37 / 282$ (13.1) were the most diagnosed AIDS defining illnesses. Sixteen patients on care died and 30 were lost to follow up. Overall the clinical management was poorly performed. ART services can successively be provided at health centre level and encourages HIV-infected persons to seek care. However, clinicians need regular clinical mentorship and supportive supervision.
\end{abstract}

Keywords: HIV, AIDS, ART, management, health centre, Tanzania

\section{Introduction}

Tanzania HIV/AIDS care and treatment services were initially focused at District and other high level health facilities $(\mathrm{MoH}, 2005)$. Health centres were left out due to several reasons including lack of qualified clinical staff, lack of adequate infrastructure and supply logistics problems (Kober \& van Damme, 2004; Wyss, 2004; Katabira \& Oelrichs, 2007). However, many patients from remote rural areas experienced difficulties in accessing care and treatment from district hospitals. The majority of patients in rural areas travel long distances to reach district hospitals and endure long waiting times in queues at the clinics (Hardon et al., 2007). As a result, patients fail to follow routine appointments and failed to adhere to appointment schedules for ART

\footnotetext{
* Correspondence: Dr. Idindili Boniphace; E-mail: boniphace.idindili@unibas.ch or idindili@yahoo.co.uk
} 
treatment. Tanzania National AIDS Control Programme (NACP) challenged with the rapid increase of AIDS patients in district and referral hospitals recognized the need to establish ART services at health centres and other selected lower health facilities aimed at improving access and adherence to ART, reduction of loss to follow up and stigma ( $\mathrm{MoH} 2005)$. Consequently, NACP and Non-Governmental Organizations (NGO) implemented a regionalization approach aimed at increasing the ART scaling-up process to cover the entire country. Each NGO was assigned specific regions to implement ART services and related programmes. A national ART accreditation protocol was developed and used to assess health care facility infrastructure capacity, available human resources and potential volume of HIV/AIDS patients. Through this process Chalinze Health Centre was accredited to provide ART service in May 2007.

In Tanzania, a typical health centre has 15-bed capacity and is managed by an Assistant Medical Officer supported by clinical officers and assistant nurses. Laboratory services at the health centre are limited to basic haemoglobin, sputum, urine and stool microscopic examinations. Usually a health centre serves as a second level of health services referral hierarchy, followed by a dispensary. A health centre is expected to cater for a population of 50,000 people which is approximately the population of one administrative division in Tanzania.

Concerns have been raised about the quality of ART services delivered in health centres due to inadequate human resource capacity and lack of laboratory facilities for monitoring CD 4 + T-lymphocytes testing (Kober \& van Damme, 2004; van Damme et al., 2006; Katabira \& Oelrichs, 2007). Furthermore, establishment of care and treatment clinics at lower levels of health care facilities poses challenges on the health system ability to pull enough resources (Morrison, 2002) to cope with increased logistics demands, supply chain management and supportive supervision. Such challenges can be addressed by health centre patient treatment cohort analysis. Currently there is lack of published data on health centre experiences of delivering ART services in Tanzania. This study therefore, was designed to gather experiences and share lessons learnt with other health care providers and programme implementing partners. The major objectives of this study were: (i) to describe HIV and AIDS case management at a rural health centre level in Tanzania; (ii) to assess short-term outcomes of patients attending care and treatment at a health centre; and (iii) to describe experiences and lessons learned in the process of implementing ART in a rural health centre in Tanzania.

\section{Materials and Methods}

\section{Setting}

Chalinze Health Centre is the largest health centre located in Chalinze in Bagamoyo District in Pwani Region of eastern Tanzania. The health centre is located at $80 \mathrm{~km}$ and $70 \mathrm{~km}$ from Bagamoyo District Hospital and Tumbi Special Hospital, respectively. The health centre serves inhabitants of seven villages around Chalinze with approximately a population of 300,000 people. Most residents are farmers and small scale traders and rely on health services delivered by the health centre. The HIV-1 seroprevalence in 2007 was 15\% (Bagamoyo District Report, unpubl.). 
The health centre started offering ART services in May 2007 with financial support from the US-President's Emergency Plan for AIDS Relief and technical support from Columbia University Mailman School of Public Health through International Centre for AIDS Program (ICAP). ICAP provided clinical training and mentorship, renovation of the health centre building to create rooms for ART services, procuring equipment and supplies, and establishing systems to support laboratory services and data management. The staffs at health centre involved with ART services delivery were trained for two weeks using training modules accredited by NACP. ART treatment started under the supervision and mentorship of experienced doctors and nursing officers from Tumbi Special Hospital in collaboration with ICAP staff. The ART start-up clinical team comprised of one clinical officer (with diploma in clinical medicine), two medical attendants (nurses), one pharmaceutical assistant and a data cum registry clerk. By the end of April 2009, a total of 895 adults and children were enrolled for ART services.

\section{Clinical services process}

ART services are provided daily Monday to Friday, two days in a week are dedicated to newly enrolled patients to receive counselling, clinical examination and laboratory evaluation. Laboratory services at the health centre are limited to basic haemoglobin, blood, urine, sputum and stool microscopic examinations. CD4+ T lymphocytes, haematology and biochemistry analysis were sent to Tumbi Special Hospital two times every week and results were obtained in the following week. Reasonable referral networks existed with dispensaries, village health posts, Bagamoyo District and Tumbi Regional Hospitals.

Patients starting to use ARVs are given an appointment according to treatment guidelines to be seen at the clinic after two weeks. From then on, provided there are no side effects, patients are seen and given ARVs at 4 weeks intervals. Clinical officers are mainly responsible for patients' clinical examination, treatment initiation, regimen changes and followup patient clinical evaluation. Assistant nurses provide counselling according to standardized protocols. Patients were eligible for ARVs if were symptomatic (WHO clinical stage III or IV), and have CD4+ T lymphocytes count of $<200 \mu 1$. Patients are required to meet a number of additional criteria including: completing ART counselling sessions, nominating a 'treatment assistant' to assist with adherence. The first-line ART regimen in Tanzania is a fixed-dose combination of stavudine (d4T), lamivudine (3TC) and nevirapine (NVP) (Triomune). In case of d4T- and NVP-related side effects, the respective alternatives are zidovudine (AZT) and efavirenz (EFV). ART is offered free of charge in all public facilities in Tanzania, including Chalinze health centre.

ART monitoring is done using standardized national monitoring tools which include care and ART registers. Patients are issued identification cards, upon registration, bearing demographic characteristics and dispensed ARVs regimen or cotrimoxazole and appointment date for the subsequent clinic visits. Standardized treatment outcomes are monitored every month in the patient master cards and updated each month in the ART register; thereafter quarterly reports are sent to the Ministry of Health and Social Welfare in Dar es Salaam.

\section{Data collection}


Two different methods of data collection were used; non-participant observations of health providers' performance and review of retrospective pre-ART and ART registers. Health providers' performance indicators include (i) history taking; (ii) physical examination; (iii) ordering laboratory tests; (iv) prescribing therapy; and (v) patient education. They were assessed as excellent (4), good (3), satisfactory (2), inadequate (1) or never done (0). In this study, the researcher took the role of non-participant observer in the consultation room. Verbal informed consent was obtained from patients and the attending clinicians. Observation check list was used to systematically document the clinician's actions and approaches. The check list was developed based on the National HIV and AIDS treatment guidelines (MoH 2005) and contained minimum set of actions which must be carried out for the care given to be classified as adequate quality care.

A sample of 40 patients categorized in two groups of newly enrolled (first clinic attendees) and continuing clients were conveniently selected for this assessment. This is to accommodate changes in clinical actions and performance of health providers when attending newly enrolled or continuing clients. The consultation time was defined as the time spent by the patient in the consultation room. Consultation start time was recorded at the moment the patient enters the clinician's room and consultation end time was recorded immediately after the patient exits the consultation room. The concern, however, is that people who know that they are being observed may change their behaviour. This challenge was addressed by making observation to last long enough (2 months), thereby making clinicians to eventually revert to their normal behaviour.

Data collected from pre-ART and ART registers reviews included demographic characteristics, place of HIV diagnosis, signs and symptoms at first enrolment to the clinic, laboratory results, treatment regimen start and stop dates and the reason for change, and reasons for treatment termination. Patients case report forms (CT2) which records patients demographic and clinical parameters, facility and patient registration codes and clinic visit dates were reviewed.

\section{Data analysis}

Data was entered into Microsoft Access, standardized quality control checks were done for completeness and duplications. Missing data from registers reviews was traced back and extracted from other hospital registers at reception and or laboratory records. Data analysis was done using Stata Intercooled version 9. The observation scores were compiled for each clinical process and the proportion of cases in which it was executed. A minimum set of actions were set according to the category of the patients (new or follow up case) all of which must be carried out for a case to have been handled with adequate quality of care. Mortality rate was calculated using Kaplan-Meir method (survival probabilities). The cumulative personal years of follow-up was calculated from the date of enrolment to cut-off point of follow-up or lost to follow-up or death of the pre-ART or ART patients. Lost to follow-up was defined as three or more consecutive months since last scheduled appointment. Comparisons of clinical manifestations was done using p-value calculated using Pearson Chi square test. A t-test was used to compare quantitative variables.

Ethical clearance 
The study received scientific and ethical approval from the Muhimbili University of Health and Allied Sciences Research Ethics Review Board (Ref.No.MU/RP/AEC/Vol.XII/58). Permission to carry out this study at the Health Centre was granted by the respective region, district and health centre authorities. Informed consent for participation was obtained from patients using Kiswahili language.

\section{Results}

Over a period of about 25 months, a total of 895 patients were attended at Chalinze Health Centre. Of these, 611 (68.3\%) were on care (pre-ART) and $284(31.7 \%)$ were on ART. Thirty (4.9\%) patients on care and $55(19.4 \%)$ on ART were lost to follow-up during the study period. The quarterly cumulative numbers of patients enrolled and retained into care and ART are shown in Error! Reference source not found..

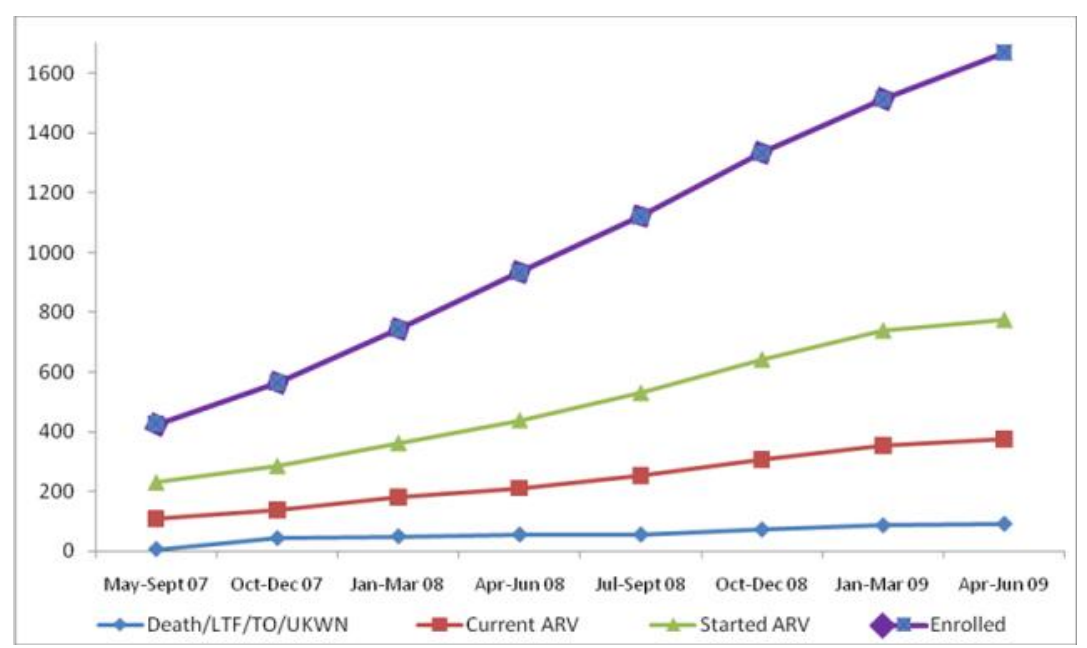

Figure 1: Cumulative number of patients enrolled, May 2007 - June 2009

Keys: LTF=lost to follow up, 2: TO=Transfer out, 3:UKWN=Unknown

Majority of patients were adults' aged between 25 to 45 years. Only 27/611 (4.4\%) pre ART and $16 / 284(5.6 \%)$ ART children were enrolled during the study period. Majority of the patients were females $622 / 895(69.5 \%)$, males had significantly $(P=0.001)$ higher mean weight compared to females (mean 53.3 and 46.3 respectively). Most patients reported to be working $605 / 895(67.6 \%)$ and $281 / 895(31.4 \%)$ were ambulatory. At presentation to the clinic, majority of patients 550/895 (61.5\%) had CD4+T-lymphocytes $\leq 350$ and 531/895(59.3\%) in WHO clinical stages III/IV. Sixteen patients on care died at different times during the study period, contributing to mortality rate of 2.13 (95\%CI 1.31 to 3.48) per 100 patient-years, and 23/284 (8.1\%) ART patients died, resulting onto mortality rate of 0.042 (95\%CI 0.01-1.01) (Table 1). Some $63.9 \%(152 / 238)$ of patients on ART had CD4+T cells $<200 / \mu 1$. 
Table 1: Characteristics of HIV patients enrolled for ART services, May 2007-June 2009

\begin{tabular}{|c|c|c|c|}
\hline Characteristics & All patients $(\mathrm{N}=895)$ & CARE patients $(\mathrm{N}=611)$ & ART-patients $(\mathrm{N}=284)$ \\
\hline \multicolumn{4}{|l|}{ Age group (years) n (\%) } \\
\hline$\leq 24$ & $133(14.8)$ & $98(16.0)$ & $35(12.3)$ \\
\hline $25-34$ & $305(34.1)$ & $212(34.7)$ & $93(32.8)$ \\
\hline $35-45$ & $315(35.2)$ & $208(34.0)$ & 107(37.8) \\
\hline$>45$ & $142(15.8)$ & $93(15.2)$ & $49(17.2)$ \\
\hline \multicolumn{4}{|l|}{ Sex $n(\%)$} \\
\hline Female & $622(69.5)$ & $433(70.8)$ & $189(66.4)$ \\
\hline Male & $273(30.5)$ & $178(29.2)$ & $95(33.6)$ \\
\hline \multicolumn{4}{|l|}{ Weight, mean(SD) kg } \\
\hline Female & $46.3(1.5)$ & $49.2(1.2)$ & $43.6(1.5)$ \\
\hline Male & $53.3(4.5)$ & $51.4(1.3)$ & $45.1(1.8)$ \\
\hline \multicolumn{4}{|l|}{ Functional status n (\%) } \\
\hline Working & $605(67.6)$ & $424(69.4)$ & 181(63.9) \\
\hline Ambulatory & $281(31.4)$ & 187(30.6) & $94(33.2)$ \\
\hline \multicolumn{4}{|l|}{ CD4+T Lymphocytes } \\
\hline$\leq 350 / \mu 1$ & $550(61.5)$ & $324(53.1)$ & $226(79.6)$ \\
\hline$>350 / \mu \mathrm{l}$ & $345(38.5)$ & $287(46.9)$ & $58(20.4)$ \\
\hline \multicolumn{4}{|l|}{ WHO clinical stage } \\
\hline I\& II & $364(40.7)$ & 291(47.6) & $73(25.7)$ \\
\hline III\& IV & $531(59.3)$ & $320(52.4)$ & $211(74.3)$ \\
\hline Haemoglobin, mean(SD) & $10.9(2.8)$ & $10.8(2.7)$ & $10.9(2.9)$ \\
\hline $\begin{array}{l}\text { Mortality rate per100 } \\
\text { patient-years }(95 \% \mathrm{CI})\end{array}$ & & $2.13(1.31-3.48)$ & $0.04(0.01-1.01)$ \\
\hline
\end{tabular}

A wide range of signs and symptoms occurred and were recorded at enrolment. Patients whose CD4+T-lymphocytes counts were low had more symptoms and signs than those with relatively higher counts. The frequency of symptoms significantly decreased with increasing CD4 counts. However, numbness and parotid enlargement were not related to patient level of CD4+Tlymphocytes counts. Genital discharge had a borderline significant association $(P=0.066)$. Many patients reported weight loss, skin itching or rashes which was followed by fever, body weakness and productive cough. Chronic diarrhoea was found in only $5.2 \%$ (47/895) of the patients and was significantly related to CD4+T-lymphocytes counts levels $(P=0.02)$ (Table 2).

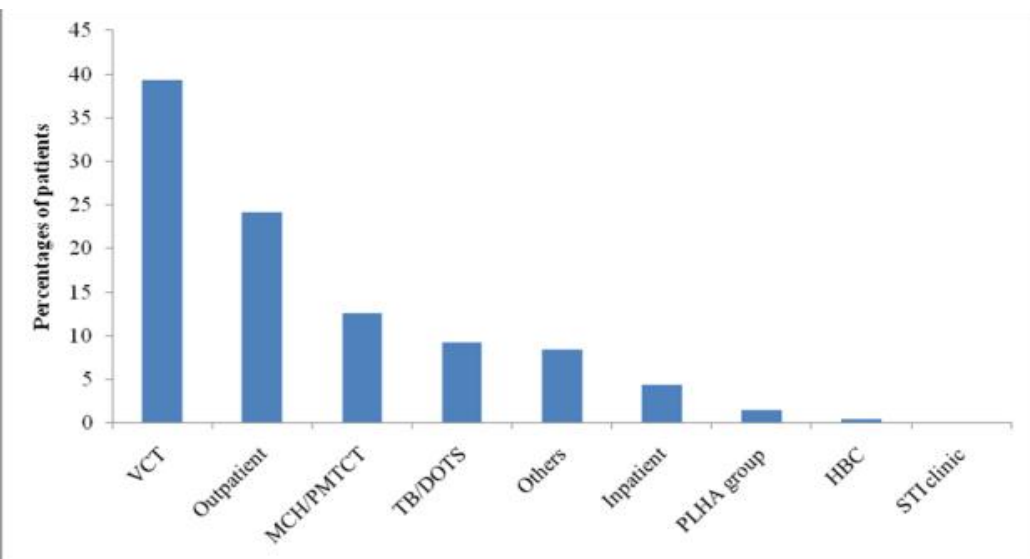

Figure 2: Place of HIV test at Chalinze Health Centre $(\mathrm{N}=895)$ 
Majority of patients enrolled for care received HIV test from Voluntary Counselling and testing centres (VCT) (39.4\%), followed by outpatient (24.2\%) and MCH/PMTCT (12.6\%) (Figure 2). Patients at the outpatient, inpatient and Tuberculosis units were tested through provider initiated testing and counselling program. Surprisingly, no patients were HIV-tested at sexually transmitted infection clinic (STI clinic) despite of training all clinical staff at the centre in provision HIV testing.

Table 2: Clinical manifestations by CD4+T-Lymphocyte counts of 895 patients with HIV and AIDS

\begin{tabular}{|c|c|c|c|c|}
\hline \multirow[t]{2}{*}{ Clinical feature } & \multicolumn{4}{|c|}{ CD4+T-Lymphocytes counts } \\
\hline & $<100(\mathrm{~N}=361)$ & $100-200(\mathrm{~N}=227)$ & $>200(\mathrm{~N}=307)$ & P value* \\
\hline Weight loss & $\mathbf{N}(\%)$ & $\mathbf{N}(\%)$ & $\mathbf{N}(\%)$ & \\
\hline Skin itching/rashes & $114(31.5)$ & $59(25.8)$ & $58(18.8)$ & 0.001 \\
\hline Fever & 107(29.6) & $45(19.9)$ & $58(18.8)$ & 0.002 \\
\hline Body weakness & $101(28.0)$ & $41(18.2)$ & $42(13.8)$ & $<0.001$ \\
\hline Productive cough & $93(25.8)$ & $34(14.8)$ & $45(14.8)$ & $<0.001$ \\
\hline Headache & $75(20.7)$ & $29(12.6)$ & $29(9.6)$ & $<0.001$ \\
\hline Numbness & $69(19.0)$ & $39(17.1)$ & $43(14.1)$ & 0.212 \\
\hline Nausea and vomiting & $40(11.1)$ & $13(5.7)$ & $14(4.7)$ & 0.003 \\
\hline Dry cough & $43(11.8)$ & $26(11.3)$ & $23(7.5)$ & 0.137 \\
\hline Parotid enlargement & $37(10.2)$ & $22(9.9)$ & $22(7.3)$ & 0.356 \\
\hline Chronic diarrhoea & $28(7.8)$ & $9(3.8)$ & $10(3.4)$ & 0.021 \\
\hline Dysphagia & $25(7.0)$ & $6(2.8)$ & $13(4.2)$ & 0.052 \\
\hline Abdominal pain & $68(18.9)$ & $23(10.2)$ & $14(4.7)$ & $<0.001$ \\
\hline Oral candidiasis & $61(16.9)$ & $16(6.9)$ & $16(5.1)$ & $<0.001$ \\
\hline Pallor & $42(11.7)$ & $6(2.8)$ & $10(3.2)$ & $<0.001$ \\
\hline Dermatitis & $19(5.3)$ & $10(4.4)$ & $7(2.2)$ & 0.139 \\
\hline Genital discharge & $17(4.6)$ & $6(2.8)$ & $5(1.7)$ & 0.066 \\
\hline
\end{tabular}

${ }^{*} \mathrm{P}$ - value calculated using Pearson Chi-square test for association

Overall 282 cases of AIDS defining illnesses (ADIs) were diagnosed and treated during the study period. The most frequent ADIs cases were papular pruritic eruptions 98/282 (34.8\%), Tuberculosis 86/282 (30.5\%) and oesophageal candidiasis 37/282 (13.1\%). Less common ADIs were Herpes zoster 16/282 (5.7\%), HIV encephalopathy 16/282 (5.7\%), HIV wasting syndrome $15 / 282$ (5.3\%), Kaposi's sarcoma 11/282 (3.9\%) and pneumocystis pneumonia 3/282 (1.1\%) (Figure 3).

Clinicians are trained to use three methods to evaluate HIV/AIDS patients' eligibility to start ART: WHO clinical staging, CD4+T-cells count or combination of these two. About 45.7\% of patients were evaluated using CD4+T-cells counts while WHO clinical staging only was used in $39.1 \%$ of patients, and both methods were applied in $15.2 \%$ of patients. The average consultation time was 4 minutes. Seventy-five percent of the consultations lasted less than 5 minutes and none more than 10 minutes. All consultations were characterized by inadequate history taking and lacked explanations of the disease, prescription and or the ordered laboratory investigations. Physical examination in terms of inspection and palpation head to toe was performed in 39\% of the patients in a sitting position and $6 \%$ were asked to lie on examination bed. Unless the patient volunteered to show a lesion on the body, most often clinicians never solicited for abnormalities. Most prescriptions were repetitions of the previous 
medications and not based on the diagnosis of the day. Occasionally patients would remind the clinician to prescribe additional medicines for the new complaint.

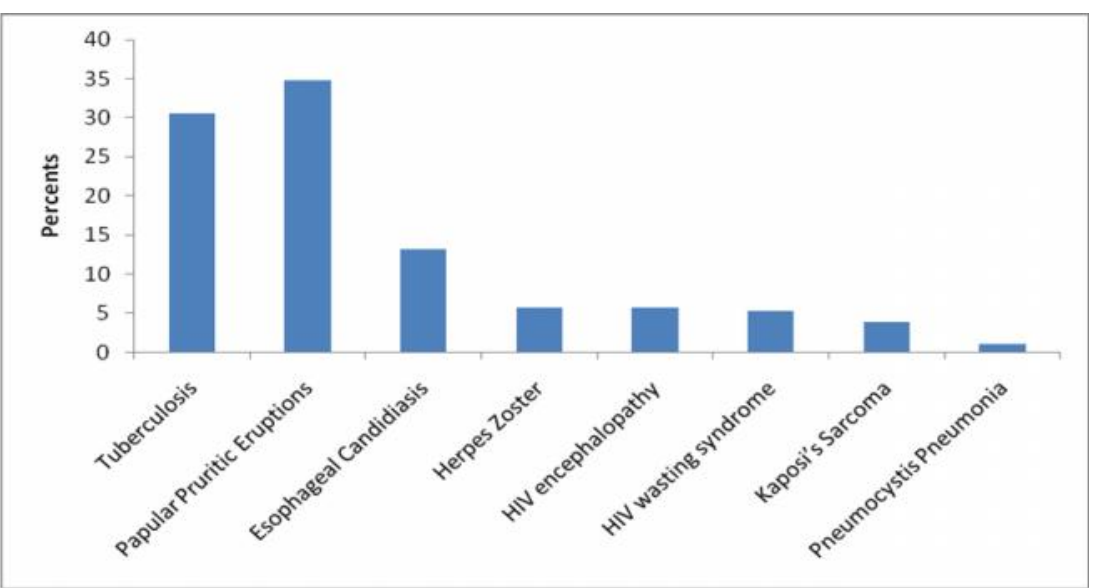

Figure 3: AIDS-defining illnesses treated, May 2007-June 2009 ( $\mathrm{N}=282)$

\section{Discussion}

Our study presents quantitative and qualitative findings; the qualitative findings were enlisted through observation of health care providers but did not collect data on patients' perceptions of the services provided and observations were done by one observer. We found that clinical management was poorly performed, marked with inadequate physical examination, inaccurate history taking and short consultation times. Despite expectations that clinical practice could be improved after ART training, we did not find any evidence to support such hypothesis. Similar practices have been reported by studies conducted in primary health care facilities in Tanzania in relation to malaria case management (Gilson et al., 1993; Nsimba et al., 2003). Clinical mentorship and regular supportive supervision programmes need to be strengthened in order to shape the clinical practice in peripheral health care facilities.

At Chalinze health centre we found an excellent referral system networks used to access ART services by patients from nearby dispensaries and the transportation of samples to Tumbi Special Hospital laboratory. The report turn-around time of the samples was seven days. Similar referral mechanisms can be established in any sites linking HIV-infected patients with care and treatment centres.

The large number of patients enrolled for HIV/AIDS care and treatment at Chalinze health centre within the short period of 25 months is alarming. However, it may be the result of increased awareness of ART health benefits, decreased HIV stigma due to ART availability close to patients' homes and the high burden of HIV/AIDS among rural population (Posse et al., 2008). HIV/AIDS associated mortality rates in rural areas of Tanzania is known to be more than 15 times compared to HIV-negative deaths rates (Urassa et al., 2001). ART services availability within short distances encourages patients who were to travel for long distances or even days to seek ART care and treatment. The proximity to services, therefore, motivates entry to care and encourages retention in care. 
Our findings point to already established facts that, it is possible to provide ART services in rural resource limited settings using existing health care workers after up-grading their skills (Wyss, 2004). Despite of the weakness and short comings documented by this study, clinic attendance was growing steadily, an indication of high demand for services and some degree of patients' satisfaction was noted with the services rendered. Patients studied experienced numerous signs and symptoms whereby cough, fever and non-specific skin rashes were the most frequently reported ones. It is important to note from this study that cough (dry and productive) is becoming more frequent sign of AIDS overtaking chronic diarrhoea. Profound weight loss, chronic diarrhoea and prolonged fevers were noted to mark major signs and symptoms of AIDS in the pre-ARVs era (Piot et al., 1984; WHO, 1987). In addition, pain in terms of headache, abdominal pain and burning or burning/tingling sensations, when both symptoms are considered across the pain trajectory becomes among the most frequently reported symptoms. Pain has been reported in other settings as one of the most frequent symptoms (Sukati et al., 2005; Bhengu et al., 2009). This finding suggests that pain management must be prioritized by clinicians caring for HIV/AIDS patients.

The number of children enrolled was rather lower than the $12 \%$ programme set target. However, diagnosis and provision of ART in HIV-infected children is more complex than in adults. Diagnosis of exposed infants up to 18 months of age requires HIV-p24, RNA or DNA polymerase chain reaction tests (Lyamuya et al., 1996). And also CD4+T cell percentage is required instead of absolute $\mathrm{CD} 4+\mathrm{T}$ cell count for older children which were not available during the study period. Therefore enrolment of children was only based on clinical manifestation and HIV antibody positive tests. It is likely that many HIV-infected children might have been missed. The ongoing implementation of early infant diagnosis using DNA polymerase chain reaction and the family centred programme are expected to increase enrolment of children into ART.

A remarkable achievement was observed through the relatively high proportions of patients received HIV-test at outpatient unit, tuberculosis unit and some from the inpatient unit of the health centre. These findings signify the implementation of provider-initiated testing and counselling (PITC), a complement to VCT. PITC was initiated with the aim of increasing uptake of HIV testing, improve access to health services for people living with HIV, and create new opportunities for HIV prevention at the time when Chalinze had only one stand alone VCT centre. PITC is implemented using the Opt-out approach (WHO, 2007) involving the health care provider specifically recommending an HIV-test to patients attended at any of the health centres' units. Then pre-test information is provided, followed by HIV test performed in the usual way unless the patient declines to be tested.

Many studies have recorded high mortality rates in the first three months in patients who were initiated on ART with CD4 <200/ $\mu$ l (Jerene et al., 2006). In contrast, in our study, none of the patients on ART died during the study period, despite of the majority started ART with CD4 $<200 / \mu l$. The possible explanation on the lack of early mortality is likely to be due to the lower prevalence of severe anaemia in this population. Severe anaemia (haemoglobin $<8 \mathrm{~g} / \mathrm{dl}$ ) is known to be correlated with high mortality rates in AIDS patients (Langford et al., 2007). In this study setting WHO clinical staging was expected to be used more frequently than CD4+T cell count in deciding ART initiation. However, due to unsatisfactory history taking and physical 
examination practices, clinicians had to wait for $\mathrm{CD} 4+\mathrm{T}$ cell counts results and delayed to initiate patients on ART. Such delays might have contributed to the deaths of patients on care.

Papular pruritic eruptions (PPE) were the most frequently diagnosed AIDS defining illness in this population. Dermatological manifestations remain a major issue for those living with HIV because skin manifestations are often disfiguring and stigmatizing. The characteristic of skin problems in HIV-infected individuals clearly indicates immunosuppression and have accompanied AIDS from early days of the epidemic (Piot et al., 1984; WHO, 1987). PPE is a cutaneous marker of advanced HIV-infection and is known to be uncommon in immunecompetent persons (Resneck et al., 2004; Eisman, 2006). Kaposi's sarcoma and Herpes zoster are companions of skin problems in AIDS patients. Although, these disease conditions occur in low frequencies but are always present in HIV-patient cohorts and are associated with severe immunodeficiency (Goedert et al., 1998). Early detection and treatment have been associated with long term survival of patients (Eisman, 2006). Thus strategies aimed at active case finding of Kaposi's sarcoma and PPE need to be developed and clinicians should be alert in assessment and initiation of interventions for these problems.

Tuberculosis remains common ADIs in HIV and AIDS patients, and is an early opportunistic disease in the course of HIV-infection which carries the highest case fatality rate (Dye et al., 1999; Corbert et al., 2003). At Chalinze health centre tuberculosis diagnosis rely entirely on clinical suspicion and sputum examination, but still about one-third of ADIs cases were due to tuberculosis. This finding indicates that more tuberculosis cases can be diagnosed in this population if standardized tuberculosis screening tools and chest X-ray examination are added to the sputum microscopy examination. Intensified case detection and contact tracing need to be instituted in tandem with community based prevention measures.

The potential limitations of this study include the fact that patients were not interviewed to solicit their views on the quality of care provided. Despite of the aforementioned limitation, we have highlighted important findings which provide confidence to programme managers and clinicians that health centres can provide ART services. In addition health centre based ART services improves access to many HIV/AIDS patients in rural areas. In conclusion, the successes and the weaknesses indicated by this study are the lessons learned and are essential references in the course of HIV/AIDS scaling-up process in rural health centres. Proper linkage and referral network of health centre ART clinics with nearby diagnostic facilities is essential to ensure quality health care in rural settings. Supportive supervision and clinical mentorship could be established simultaneously with the initiation of ART services in health centres.

\section{Competing interest}

No competing interests expressed by authors.

\section{Acknowledgements}

The authors express their sincere gratitude to patients who gave their time to participate in this research. We thank all staff of Chalinze health Centre where the study was conducted. We also thank the District Executive Director of Bagamoyo District for granting us permission to use the 
Health centre facilities to conduct this study. Thanks to Prof. Melkizedeck Leshabari for his advice and inputs. We extend special thanks to all research assistants and Ms Rehmant Songoro for support in collection and entry of data. We also acknowledge the support of the Swiss Tropical and Public Health Institute for their continuous support at all the stages of this study. The study received funding from International Ford Scholarships and the Swiss Tropical and Public Health Institute, an associated institute of the University of Basel.

\section{References}

Bhengu, .BR., Ncama, B.P., McInerney, P.A., Wantland, D.J., Nicholas, P.K., Corless, I.B., McGibbon, C.A., Davis, S.M., Nicholas, T.P. \& Ros, A.V. (2009) Symptoms experienced by HIV-infected individuals on antiretroviral therapy in KwaZulu-Natal, South Africa. Applied Nursing Research 24, 1-9.

Corbett, E.L. Watt,C.J., Walker, M. Maher, D., Williams, B.G., Raviglione, M.C. \& Dye, C. (2003) The growing burden of tuberculosis: global trends and interactions with the HIV epidemic. Archives of Internal Medicine 163, 1009.

Dye, C., Schele, S., Dolin, P., Pathania, V. \& Raviglione, M.C. (1999) Global burden of tuberculosis: estimated incidence, prevalence, and mortality by country. Journal of American Medical Association 282, 677.

Eisman, S. (2006) Pruritic papular eruption in HIV. Dermatologic Clinics 24, 449-457.

Gilson, L., Kitange, H. \& Teuscher, T. (1993) Assessment of process quality in Tanzanian primary care. Health Policy 26, 119-139.

Goedert, J., Cote, T., Virgo, P., Scoppa, S.M., Kingma, D.W., Gail, M.H. \& Jaffe, E.S. (1998) Spectrum of AIDS-associated malignant disorders. Spectrum 1, 2.

Hardon, A.P., Akurut, D., Comoro, C., Ekezie, C., Irunde, H.F., Gerrits, T., Kglatwane, J., Kinsman, J., Kwasa, R., Maridadi, J., Moroka, T.M., Moyo, S., Nakiyemba, A., Nsimba, S., Ogenyi, R., Oyabba, T., Temu, F. \& Laing, R. (2007) Hunger, waiting time and transport costs: time to confront challenges to ART adherence in Africa. AIDS Care 19, 658-665.

Jerene, D., Endale, A., Hailu, Y. \& Lindtjorn, B. (2006) Predictors of early death in a cohort of Ethiopian patients treated with HAART. BMC Infectious Diseases 6, 136.

Katabira, E.T. \& Oelrichs, R.B. (2007) Scaling up antiretroviral treatment in resource-limited settings: successes and challenges. AIDS 21 (Suppl 4), S5-S10.

Kober, K. \& van Damme, W. (2004) Scaling up access to antiretroviral treatment in southern Africa: who will do the job? The Lancet, 364, 103-107.

Langford, S.E., Ananworanich, J. \& Cooper, D.A. (2007) Predictors of disease progression in HIV infection: a review. AIDS Research and Therapy 4, 11.

Lyamuya, E., Bredberg-R.A., Massawe, A., Urassa, E., Kawo, G., Msemo, G., Kazimoto, T., Vostborn, A., Karlsson, R. \& Mhalu, F.(1996) Performance of a modified HIV-1 p24 antigen assay for early diagnosis of HIV-1 infection in infants and prediction of motherto-infant transmission of HIV-1 in Dar es Salaam, Tanzania. Journal of Acquired Immune Deficiency Syndrome 12, 421. 
$\mathrm{MOH}$ (2005) National Guidelines for the Clinical Management of HIV and AIDS. $2^{\text {nd }}$ ed. Ministry of Health Tanzania, United Republic of Tanzania April 2005.

Morrison, J.S. (2002) Expanding antiretroviral treatment in developing countries creates critical new challenges. Centre for Strategic and International Studies HIVIAIDS Task Force Report.

Nsimba, S.E., Massele, A.Y., Eriksen, J., Gustafsson, L.L., Tomson, G. \& Warsame, M. (2002) Case management of malaria in under-fives at primary health care facilities in a Tanzanian district. Tropical Medicine E International Health 7, 201-209.

Piot, P., Taelman, H., Minlangu, B., Mbendi, N., Ndangi, K., Kambayi, K., Bridts, C., Quinn, T.C., Feinsod, F.M. \& Wobin, O. (1984) Acquired immunodeficiency syndrome in heterosexual population in Zaire. Lancet 324, 65-69.

Posse, M., Meheus, F., Asten, H., Ven, A. \& Baltussen, R. (2008) Barriers to access to antiretroviral treatment in developing countries: a review. Tropical Medicine $\mathcal{E}$ International Health 13, 904-913.

Resneck Jr, J.S., Van, B.M., Furmanski, L., Oyugi, J., LeBoit, P.E., Katabira, E., Kambugu, F., Maurer, T., Berger, T. \& Pletcher, M. J. (2004) Etiology of pruritic papular eruption with HIV infection in Uganda. Journal of American Medical Association 292, 2614-2621.

Sukati, N.A., Makoa, E., Makoae, L.N., Seboni, N.M. \& Human, S. (2005) HIV/AIDS symptom management in Southern Africa. Journal of Pain and Symptom Management 29, 185-192.

Urassa, M., Boerma, J., Isingo, R., Ngalula, J., Ngweshemi, J., Mwaluko, G. \& Zaba, B. (2001) The impact of HIV/AIDS on mortality and household mobility in rural Tanzania. AIDS 15, 2017-2023.

van Damme, W., Kober, K. \& Laga, M. (2006) The real challenges for scaling up ART in subSaharan Africa. AIDS 20, 653-656.

WHO (1987), Acquired Immunodeficiency Syndrome (AIDS), 1987 revision of CDC/WHO case definition of AIDS. Weekly Epidemiological Record 1988; 634-637.

WHO (2007), HIV/AIDS Programme Strengthening Health Services to Fight HIV/AIDS 2007, Guidance on Provider-initiated HIV Testing and Counseling in Health facilities.

Wyss, K. (2004) An approach to classifying human resources constraints to attaining healthrelated Millennium Development Goals. Human Resources for Health 2, 6. 